\title{
On the Throughput Characteristics of Utility-based Fair Scheduling
}

\author{
Erwu Liu and Kin K. Leung \\ Department of Electrical and Electronic Engineering \\ Imperial College \\ London, United Kingdom \\ \{erwu.liu, kin.leung@imperial.ac.uk\}
}

\begin{abstract}
The proportional fair scheduling (PFS) problem is studied in the paper. PFS is considered an attractive bandwidth allocation criterion in wireless networks for supporting high resource utilization while maintaining good fairness among network flows. The most challenge of a PFS problem is the lack of an analytic expression. By rigorously mathematical derivation, we obtain a closed-form expression for the throughput of PFS in Rayleigh fading environment. The theoretical results are compared with those from simulations. The derived model is shown to provide a high accuracy in evaluating the throughput of the PFS algorithm in Rayleigh fading networks. In particular, the expression presented here will provide great help for the system design of a PFS capable network. Moreover, compared with existing analytical results on PFS, our expression is more general in that we do not require the i.i.d relationship among nodes in our derivation.
\end{abstract}

Keywords-proportional fair scheduling, gaussian approximation, rayleigh fading

\section{INTRODUCTION}

Among various researches on wireless scheduling, the proportional fair scheduling (PFS) algorithm has been widely conceived an attractive solution as it provides a good compromise between the maximum throughput and node fairness by exploiting multi-user diversity and game-theoretic equilibrium in fading wireless environment.

Since its first presence [1], there has been substantial interests in the PFS algorithm in wireless networks (see [2, 3, 4] and the references therein). Though the PFS algorithm has garnered so much attention and currently implemented in $3 \mathrm{G}$ wireless network for high data rate delay-tolerant services [5], most existing results are obtained from computer simulations. To the best of our knowledge, limited mathematical analysis on the throughput of the PFS [7-11] are obtained, either assuming a simplified form of the original PFS preference metric or assuming simple linear or logarithm rate models. In the linear model, the feasible rate is linearly proportional to the signal to interference-plus-noise ratio (SINR), while in the logarithm model there is a logarithmic relationship between the SINR and the feasible rate. These two models have their merits as they greatly simplify the mathematical analysis of PFS. For example, [7] [8] and [12] used the linear rate model, while a recent paper [10] used the logarithm rate model, to analyze PFS. The assumption of linear or logarithm rate model is a reasonable modeling convention. However, when examining throughput performance, it does not seem entirely satisfactory to assume such simplified models. Works by Telatar [13] and Smith et al. [14] suggested that the feasible rate over Rayleigh fading channels can be better modeled by a Gaussian distribution with surprisingly high accuracy.

Moreover, most researches are assuming some kinds of i.i.d relationship among users/nodes in their derivations [8] [10]. For example, [10] assumes that node $k$ 's SINR $S_{k}=c_{k} \times C(\forall k)$, where $c_{k}$ is a node-related constant and $C$ is a distribution independent of all nodes (i.e., $C$ is i.i.d for all nodes). Undoubtedly, these assumptions limit the use of existing theoretical results on PFS.

In all, our goal is to provide formal, yet intuitive formulation which applies to more general scenarios without the limitations above, i.e., our analysis will not require the i.i.d relationship among nodes and also Gaussian approximation (GA) method is used to accurately model the feasible rate.

The rest of the paper is structured as follows. Section II presents the system model and the problem formulation. The mathematical analysis is conducted in Section III. In Section IV, numerical and simulation results are presented and compared to validate the closed-form expression given in Section III followed by the conclusion in Section V.

\section{SySTEM MODEL AND PROBLEM FORMULATION}

Consider a single-cell system shown in Fig. 1, $N$ mobile stations (denoted as nodes $m_{1}, m_{2}, \ldots$, and $m_{N}$ ) are randomly located within the cell served by a base station (BS).

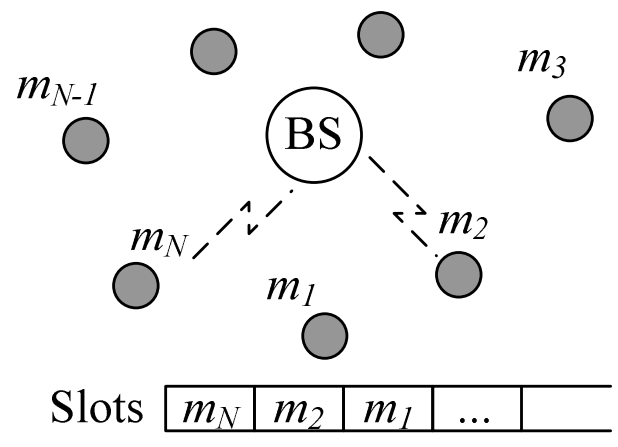

Figure 1. Single-hop wireless network 
Consider the problem where these $N$ nodes wishing to transmit data to the base station, and the rates of transmission are randomly varying. Time is divided into small scheduling intervals called slots. Until further notice, in each slot only one node is chosen to transmit. The selection of the node to schedule is based on a balance between the current possible rates and fairness. The proportional fair scheduling (PFS) [1, 2, $9,10]$ performs this by comparing the ratio of the feasible rate for each node to its average throughput tracked by an exponential moving average, which is defined as the preference metric. The node with the maximum preference metric will be selected for transmission at the next scheduling slot. This is described mathematically as follows. The end of slot $n$ is called time $n$. In next time slot $n+1$, the instantaneous data rate of node $j$ will be $R_{j}[n+1]$. Its $k$-point moving average throughput up to time $n$ is denoted by $r_{j ; k}[\mathrm{n}]$, and the preference metric (PF metric) by $M_{j ; k}[n+1]=R_{j}[n+1] / r_{j ; k}[n]$.

Node $i=\arg \max _{j} M_{j ; k}[n+1]=\arg \max _{j} R_{j}[n+1] / r_{j ; k}[n]$ is chosen to transmit in next time slot $n+1$. The moving average throughput of node $j$ up to time $n+1$ of is updated by

$r_{j ; k}[n+1]=\left(1-\frac{1}{k}\right) r_{j ; k}[n]+I_{j}[n+1] \times \frac{R_{j}[n+1]}{k}$

where $I_{j}[n+1]$ is the indicator function of the event that node $j$ is scheduled to transmit in time slot $n+1$.

$I_{j}[n+1]=\left\{\begin{array}{l}1, \text { node } j \text { scheduled in slot } n+1 \\ 0, \quad \text { else }\end{array}\right.$

By introducing utility function $U_{j}=\log \left[r_{j}\right]$, Kelly [1] had proved that the sum of the user utility (user satisfaction indicator) is maximized under the PFS criteria. It is the logarithm utility maximization, the multi-user diversity gains and the possibility to schedule bad-channel-condition nodes that make the $P F$ scheduler superior to the traditional ones such as round-robin $(R R)$ and opportunistic scheduler.

As PF metric is directly related to the feasible rate $R$, for the analysis to be as accurate as possible, it is natural to assume in our analysis that the feasible rate over Rayleigh fading channels is Gaussian [14]. For single-input-single-output (SISO) case, the Gaussian approximation (GA) method in [14] reduces to the following form,

$$
\begin{gathered}
E[R]=W \int_{0}^{\infty} \log (1+\operatorname{SINR} \times \lambda) \times e^{-\lambda} d \lambda \\
\sigma_{R}^{2}=W^{2} \int_{0}^{\infty}(\log (1+\operatorname{SINR} \times \lambda))^{2} \times e^{-\lambda} d \lambda \\
-W^{2}\left(\int_{0}^{\infty} \log (1+\operatorname{SINR} \times \lambda) \times e^{-\lambda} d \lambda\right)^{2}
\end{gathered}
$$

where $W$ is the bandwidth, $E[R]$ and $\sigma_{R}$ are the mean value and the standard deviation of $R$, respectively.

\section{PFS: CLOSE-FORM EXPRESSION}

From (1), the expect value of the $k$-point moving average throughput of node $j$ up to time $n+1$ is

$$
E\left[r_{j ; k}[n+1]\right]=\left(1-\frac{1}{k}\right) E\left[r_{j ; k}[n]\right]+\frac{E\left[I_{j}[n+1] R_{j}[n+1]\right]}{k}
$$

where $E($.$) denotes the statistical average, r_{j ; k}[n]$ is the $k$-point moving average throughput of node $j$ up to time $n$

Assuming wide-sense stationary $r_{j ; k}$, we then have

$$
E\left[r_{j ; k}[n+1]\right\rfloor=E\left[r_{j ; k}[n]\right]=E\left[I_{j}[n+1] R_{j}[n+1]\right\rfloor
$$

Applying (2) to (6) yields

$$
E\left[r_{j ; k}[n]\right]=E\left[R_{j}[n+1] \mid I_{j}[n+1]=1\right] \times \operatorname{Pr}\left(I_{j}[n+1]=1\right)
$$

where $\operatorname{Pr}\left(I_{j}[\mathrm{n}+1]=1\right)$ is the average probability that node $j$ will be scheduled in time slot $n+1$.

The feasible rate $R_{j}$ is always greater than 0 . Applying Bayes's theorem, we can write (7) as

$$
\begin{aligned}
E\left[r_{j ; k}[n]\right] & =\operatorname{Pr}\left(I_{j}[n+1]=1\right) \times \int_{0}^{\infty} x f_{R_{j}}\left(x \mid I_{j}[n+1]=1\right) d x \\
& =\int_{0}^{\infty} x f_{R_{j}}(x) \operatorname{Pr}\left(I_{j}[n+1]=1 \mid R_{j}[n+1]=x\right) d x
\end{aligned}
$$

where $f_{R j}($.$) denotes the probability density function of R_{j}$.

Under the PFS criteria stated earlier,

$\operatorname{Pr}\left(I_{j}[n+1]=1 \mid R_{j}[n+1]=x\right)=\operatorname{Pr}\left(\forall i \neq j, \frac{R_{i}[n+1]}{r_{i ; k}[n]}<\frac{x}{r_{i ; k}[n]}\right)$

For statistically independent $R_{j}$ among nodes, (9) can be written in the form

$$
\operatorname{Pr}\left(I_{j}[n+1]=1 \mid R_{j}[n+1]=x\right)=\prod_{i=1, i \neq j}^{N} F_{R_{i}}\left(\frac{x}{r_{j ; k}[n]} r_{i ; k}[n]\right)
$$

By use of (1), we have

$\lim _{n \rightarrow \infty}\left\{\lim _{k \rightarrow \infty} \frac{r_{i ; k}[n]}{r_{j ; k}[n]}\right\}==\lim _{k \rightarrow \infty}\left\{\lim _{n \rightarrow \infty} \frac{\sum_{m=1}^{n} r_{i ; k}[m]}{\sum_{m=1}^{n} r_{j ; k}[m]}\right\}=\lim _{k \rightarrow \infty} \frac{A\left[r_{i ; k}\right]}{A\left[r_{j ; k}\right]}$

where $A[\cdot]$ denotes the time average.

For wide-sense stationary $r_{j ; k}$, we further assume it is firstorder ergodic, then

$\lim _{n \rightarrow \infty}\left\{\lim _{k \rightarrow \infty} \frac{r_{i ; k}[n]}{r_{j ; k}[n]}\right\}=\lim _{k \rightarrow \infty} \frac{E\left[r_{i ; k}\right]}{E\left[r_{j ; k}\right]}=\frac{E\left[r_{i}\right]}{E\left[r_{j}\right]}$

Therefore, for large $n, k,(10)$ can be written as

$$
\begin{aligned}
\operatorname{Pr}\left(I_{j}[n+1]=1 \mid R_{j}[n+1]=x\right) & =\prod_{i=1, i \neq j}^{N} F_{R_{i}}\left(\frac{x}{r_{j ; k}[n]} r_{i ; k}[n]\right) \\
& \approx \prod_{i=1, i \neq j}^{N} F_{R_{i}}\left(\frac{E\left[r_{i}\right]}{E\left[r_{j}\right]} x\right)
\end{aligned}
$$

On substitution of (13) into (8), we obtain

$$
E\left[r_{j}\right]=\lim _{n, k \rightarrow \infty} E\left[r_{j, k}[n]\right]=\int_{0}^{\infty} x f_{R_{j}}(x) \prod_{i=1, i \neq j}^{N} F_{R_{i}}\left(\frac{E\left[r_{i}\right]}{E\left[r_{j}\right]} x\right) d x
$$

where $F_{R j}($.$) is the distribution function of R_{j}$.

Assuming $R_{j}$ is Gaussian as described earlier, we have 


$$
\begin{aligned}
E\left[r_{j}\right] & =\int_{0}^{\infty} x f_{R_{j}}(x) \prod_{i=1, i \neq j}^{N} F_{R_{i}}\left(\frac{E\left[r_{i}\right]}{E\left[r_{j}\right]} x\right) d x \\
& =\int_{-}^{\infty} \frac{E\left[R_{j}\right]}{\sigma_{R_{j}}}\left(y \sigma_{R_{j}}+E\left[R_{j}\right]\right) \frac{1}{\sqrt{2 \pi}} e^{-\frac{y^{2}}{2}} \\
& \left.\times \prod_{i=1, i \neq j}^{N} F_{R_{i}}\left(\frac{E\left[r_{i}\right]}{E\left[r_{j}\right.}\right]\left(y \sigma_{R_{j}}+E\left[R_{j}\right]\right)\right) d y
\end{aligned}
$$

where $E\left[R_{j}\right], \sigma_{R j}$ are the expect value and standard deviation of $R_{j}$, respectively.

For the feasible rate determined by (3) and (4), it can be proved that,

$$
\left\{\begin{array}{l}
E\left[R_{j}\right]>E\left[R_{i}\right] \text { and } \frac{E\left[R_{j}\right]}{\sigma_{R_{j}}}>\frac{E\left[R_{i}\right]}{\sigma_{R_{i}}} \text {, if } \sigma_{R_{j}}>\sigma_{R_{i}} \\
E\left[R_{j}\right]=E\left[R_{i}\right] \text { if } \sigma_{R_{j}}=\sigma_{R_{i}}
\end{array}\right.
$$

It is well-known that, with proportional fair scheduling, node $j$ will expect higher mean throughput $E\left[r_{j}\right]$ when the mean $\operatorname{SINR}_{j}$ is increased. So, from (3) and (4), we will have higher $E\left[R_{j}\right]$ and $\sigma_{j}$ for higher $S I N R_{j}$. Using (16), we then have

$$
\frac{E\left[r_{j}\right] E\left[R_{i}\right]-E\left[r_{i}\right] E\left[R_{j}\right]}{E\left[r_{i}\right] \sigma_{R_{j}}-E\left[r_{j}\right] \sigma_{R_{i}}}<0 \text {, for } \sigma_{R_{j}} \neq \sigma_{R_{i}}
$$

\section{I. when all $\sigma_{R i}(i=1,2, \ldots, N)$ are equal.}

As $F_{R i}(x)=F_{(0,1)}\left(\left(x-E\left[R_{i}\right]\right) / \sigma_{R i}\right)$ for Gaussian $R_{i}$, where $F_{0,1}($.$) is$ zero mean, unit variance standard normal distribution function,

$$
\begin{aligned}
E\left[r_{j}\right] & =\int_{-\frac{E\left[R_{j}\right]}{\sigma_{R_{j}}}}^{\infty}\left(y \sigma_{R_{j}}+E\left[R_{j}\right]\right) \frac{1}{\sqrt{2 \pi}} e^{-\frac{y^{2}}{2}} \\
& \times \prod_{i=1, i \neq j}^{N} F_{R_{i}}\left(\frac{E\left[r_{i}\right]}{E\left[r_{j}\right]}\left(y \sigma_{R_{j}}+E\left[R_{j}\right]\right)\right) d y \\
& =\int_{-\frac{E\left[R_{j}\right]}{\sigma_{R_{j}}}}^{\infty}\left(y \sigma_{R_{j}}+E\left[R_{j}\right]\right) \frac{1}{\sqrt{2 \pi}} e^{-\frac{y^{2}}{2}} \\
& \times\left(F_{R_{i}}\left(y \sigma_{R_{i}}+E\left[R_{i}\right]\right)\right)^{N-1} d y \\
& =\int_{-\frac{E\left[R_{j}\right]}{\sigma_{R_{j}}}}^{\infty}\left(y \sigma_{R_{j}}+E\left[R_{j}\right]\right) f_{(0,1)}(y) \times\left(F_{(0,1)}(y)\right)^{N-1} d y
\end{aligned}
$$

where $f_{0,1}($.$) is zero mean, unit variance standard normal$ probability density function.

\section{II. when not all $\sigma_{R i}(i=1,2, \ldots, N)$ are equal.}

Denote $Z=\max _{i}\left[\left(E\left[r_{j}\right] E\left[R_{i}\right]-E\left[r_{i}\right] E\left[R_{j}\right]\right) /\left(E\left[r_{i}\right] \sigma_{R j}-E\left[r_{j}\right] \sigma_{R i}\right)\right]$, we will have

$$
Z \geq \frac{E\left[r_{j}\right] E\left[R_{i}\right]-E\left[r_{i}\right] E\left[R_{j}\right]}{E\left[r_{i}\right] \sigma_{R_{j}}-E\left[r_{j}\right] \sigma_{R_{i}}} \geq-\frac{E\left[R_{j}\right]}{\sigma_{R_{j}}}
$$

And,

$$
Z \leq-\max _{i}\left[-\frac{E\left[R_{i}\right]}{\sigma_{R_{i}}}\right]
$$

We can write (14) as

$$
\begin{aligned}
E\left[r_{j}\right] & =\int_{-\frac{E\left[R_{j}\right]}{\sigma_{R_{j}}}}^{Z}\left(y \sigma_{R_{j}}+E\left[R_{j}\right]\right) f_{(0,1)}(y) \\
& \times \prod_{i=1, i \neq j}^{N} F_{R_{i}}\left(\frac{E\left[r_{i}\right]}{E\left[r_{j}\right]}\left(y \sigma_{R_{j}}+E\left[R_{j}\right]\right)\right) d y+ \\
& \int_{Z}^{\infty}\left(y \sigma_{R_{j}}+E\left[R_{j}\right]\right) f_{(0,1)}(y) \\
& \times \prod_{i=1, i \neq j}^{N} F_{R_{i}}\left(\frac{E\left[r_{i}\right]}{E\left[r_{j}\right]}\left(y \sigma_{R_{j}}+E\left[R_{j}\right]\right)\right) d y
\end{aligned}
$$

Also, from (16), (17) and (19), it is easy to prove that

$$
\frac{E\left[r_{i}\right]}{E\left[r_{j}\right]}\left(y \sigma_{R_{j}}+E\left[R_{j}\right]\right) \geq y \sigma_{R_{i}}+E\left[R_{i}\right] \text {, for } y \geq Z
$$

By (19), the first integral in the right hand of (21) is not less than 0. Using $F_{R i}(a) \geq F_{R i}(b) \forall a \geq b$ together with (22) and (21), we have

$$
\begin{aligned}
E\left[r_{j}\right] & \geq \int_{Z}^{\infty}\left(y \sigma_{R_{j}}+E\left[R_{j}\right]\right) f_{(0,1)}(y) \\
& \times \prod_{i=1, i \neq j}^{N} F_{R_{i}}\left(\frac{E\left[r_{i}\right]}{E\left[r_{j}\right]}\left(y \sigma_{R_{j}}+E\left[R_{j}\right]\right)\right) d y \\
& \geq \int_{Z}^{\infty}\left(y \sigma_{R_{j}}+E\left[R_{j}\right]\right) f_{(0,1)}(y) \\
& \times \prod_{i=1, i \neq j}^{N} F_{R_{i}}\left(y \sigma_{R_{i}}+E\left[R_{i}\right]\right) d y \\
& =\int_{Z}^{\infty}\left(y \sigma_{R_{j}}+E\left[R_{j}\right]\right) f_{(0,1)}(y)\left(F_{(0,1)}(y)\right)^{N-1} d y \\
& \geq \int_{M}^{\infty}\left(y \sigma_{R_{j}}+E\left[R_{j}\right]\right) f_{(0,1)}(y)\left(F_{(0,1)}(y)\right)^{N-1} d
\end{aligned}
$$

where $M=-\max _{i}\left[E\left[R_{i}\right] / \sigma_{R i}\right](i=1,2, \ldots, N)$.

We can rewrite (18) and (23) by one single expression,

$$
\begin{aligned}
E\left[r_{j}\right] & \geq \int_{M}^{\infty}\left(y \sigma_{R_{j}}+E\left[R_{j}\right]\right) f_{(0,1)}(y)\left(F_{(0,1)}(y)\right)^{N-1} d y \\
& \geq \frac{E\left[R_{j}\right]}{N}+\int_{M}^{\infty} y \sigma_{R_{j}} f_{(0,1)}(y)\left(F_{(0,1)}(y)\right)^{N-1} d y
\end{aligned}
$$

Now we have the closed-form expression for the PFS throughput. This intuitive yet formal formula has the great practical and theoretical interests of being mathematically graceful and simple. 
(24) reveals a very interesting merit of PFS that has not been seen elsewhere in literatures: node $j$ 's throughput is solely (approximately, if we ignore the effect of different $M$ on the integral) determined by the network size together with its own channel statistics when the feasible rate is Gaussian.

Other observations can also be made from (24). First of all, as $E\left[R_{j}\right] / N$ is the mean throughput of node $j$ when using round robin (RR) scheduling, the second item in the right hand of (24) is in fact the improvement of node $j$ 's throughput when using PFS instead of RR scheduling.

Obviously, PFS will provide more benefits in severe fading environments where $\sigma_{R j}$ is large. On the other hand, PFS can be viewed as a RR scheduling algorithm in low-fading environments where $\sigma_{R j}$ is relatively small compared with $E\left[R_{j}\right]$.

Fig.2 shows that our expression given by (24) will give the PFS throughput very close to the existing one [10]. In addition, we would like to point out that the result from [10] has limitation in that it assumes node $k$ 's $\operatorname{SINR} S_{k}=c_{k} \times C(\forall k)$, where $c_{k}$ is a node-related constant and $C$ is a distribution independent of all nodes, i.e., $E\left[S_{k}\right] / \sigma_{S k}=E[C] / \sigma_{C}$ is constant for all nodes. Clearly, the theoretical expression presented here is more general as we do not require such i.i.d assumption and more accurate rate model is used in our derivation.

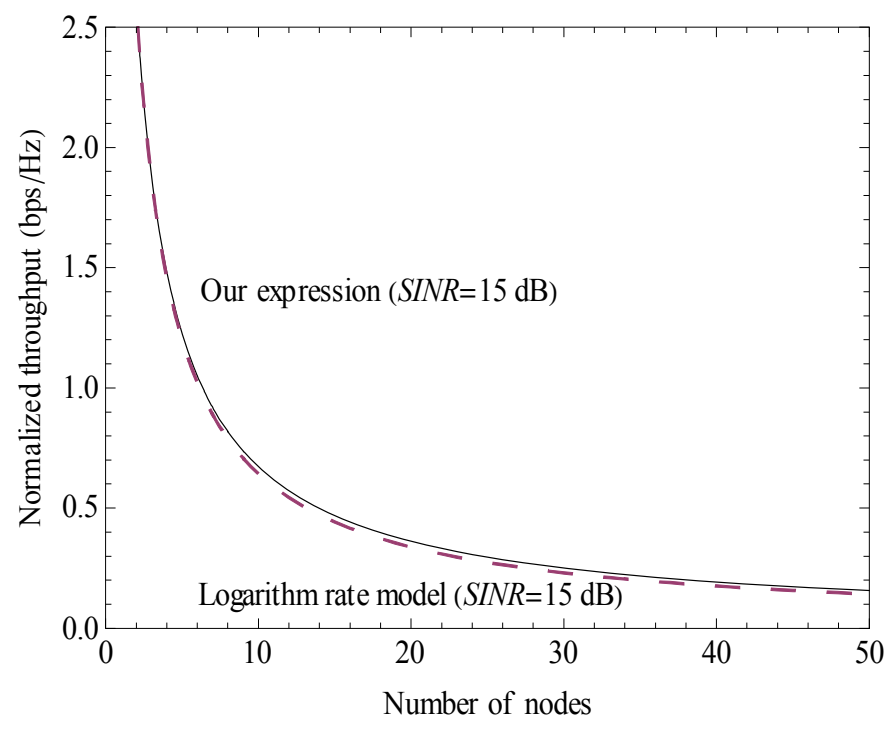

Figure 2. PFS throughput plotted from (25) (the solid line) and [10] (the dashed one)

\section{VALIDITY OF THE ANALYTIC EXPRESSION BY COMPARISON WITH SIMULATION RESULTS}

The analytic expression for the throughput of PFS is now evaluated by comparing the numerical and simulation results. In the simulation, the initial moving average throughputs of nodes are randomized and we use two different methods to model the feasible rate $R_{j}$ over Rayleigh fading channel.

Method $\boldsymbol{A}$ ) the feasible rates over Rayleigh fading channels are generated according to statically independent Gaussian distribution for different nodes, i.e., Gaussian approximation (GA) method, and
Method B) standard method, i.e., $R_{j}=W \times \log \left[1+S I N R_{j} \times\left|h_{j}\right|^{2}\right]$, where the channel gain $h_{j}$ for node $j$ is a normalized complex Gaussian random variable.

System parameters are: $W=20 \mathrm{MHz}$ bandwidth, $k=500$ for $k$-point moving average calculation, 15 nodes (node1 node15) with mean SINR equal to $2,5,5,8,10,12,15,15,16,18,20$, $22,25,25$ and $28 \mathrm{~dB}$, respectively.

For simplicity, in method $A$, we use the notation $n_{j}\left[E\left[R_{j}\right], \sigma_{R j}\right]$ to indicate the feasible rate of node $j$ has mean value $E\left[R_{j}\right]$ and standard deviation $\sigma_{R j}$ (in Mbps). Node $j$ has randomized data rate mean $E\left[R_{j}\right]$ and standard deviation $\sigma_{R j}$ determined by (3) and (4). For the given SINR, the feasible data rates of nodes under method $A$ are then characterized as:

$n_{1}[23.2,15.0] \quad n_{2}[34.3,19.6] \quad n_{3}[34.3,19.6] \quad n_{4}[47.9,23.8] \quad n_{5}[58.1,26.3]$

$\begin{array}{llllll}n_{6}[69.1,28.5] & n_{7}[86.6,31.1] & n_{8}[86.6,31.1] & n_{9}[92.7,31.9] & n_{10}[105.0,33.1]\end{array}$

$n_{11}[117.7,34.1] n_{12}[130.5,34.8] n_{13}[150,35.6] \quad n_{14}[150,35.6] \quad n_{15}[169.7,36.1]$

We plot in Fig. 3 the throughput of node7 and node8 (both with $S I N R=15 \mathrm{~dB}$ ). It is clear that the analytic result produces a accurate estimate of the PFS throughput for both simulation methods. The theoretical expression (24) is also validated by the plots that nodes of same channel statistics have the same mean throughput.

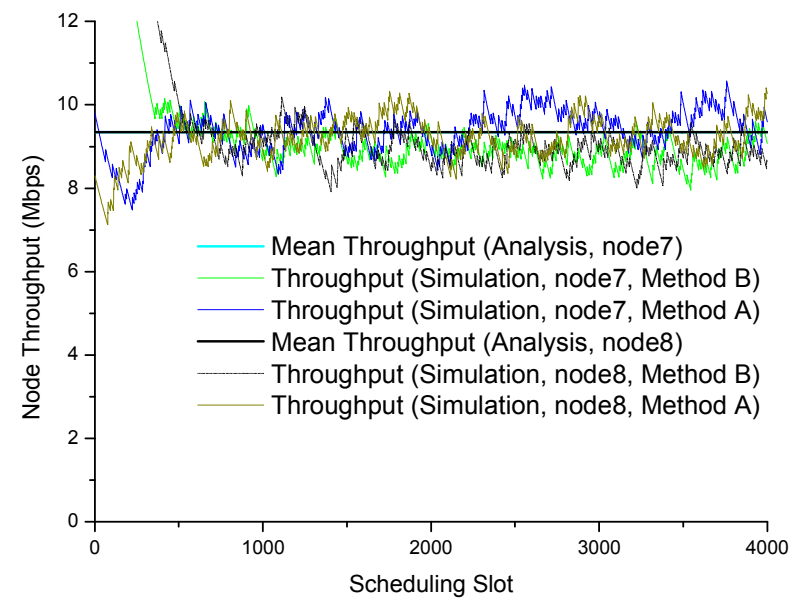

Figure 3. Accuracy of the analytic expression for node throughput

To further evaluate the theoretical formula, we plot in Fig. 4 the throughput of nodes experienced remarkably different channel conditions. Node1 has the worst channel statistics in the network, i.e., $S I N R=2 \mathrm{~dB}$, while Node15 has the best channel statistics, i.e., $S I N R=28 \mathrm{~dB}$. Once again, the validity of (24) is justified by the perfect match between the analysis and simulation results for these two extreme cases.

Both figures show that the average throughputs from simulation eventually converge to those determined by analytic expression (24). The overall conclusion from these experiments suggests the closed-form expressions presented in Section III can be used with confidence to evaluate the performance of the PFS algorithm in Rayleigh fading environment. The analytical 
results given in the paper will greatly facilitate the system design of a PFS-capable network.

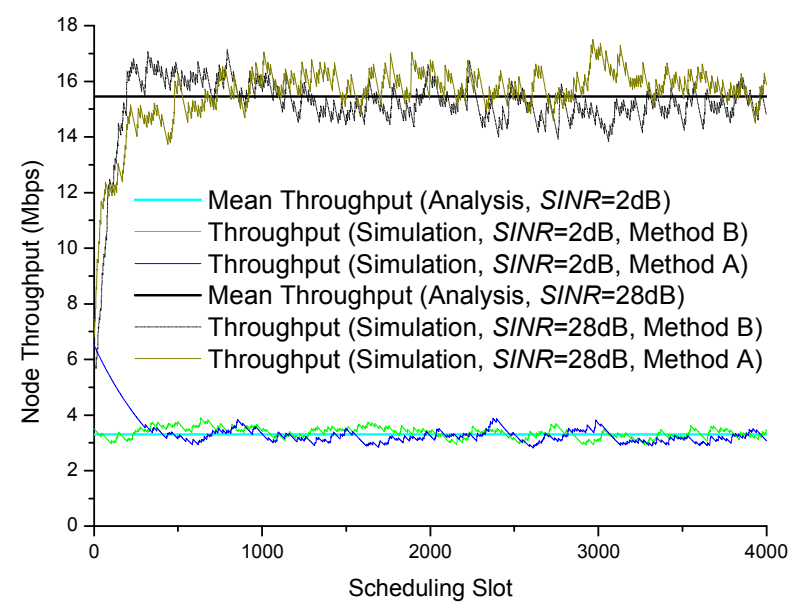

Figure 4. Accuracy of the analytic expression for node throughput

\section{CONCLUSIONS}

An intuitive yet formal analytic solution for the throughput of proportional fair scheduling in Rayleigh fading systems is developed. Comparison with simulations shows that our analysis provides an accurate estimate of the PFS throughput. Moreover, the theoretical results presented here are more general than existing ones [8] [10] in that we do not require the i.i.d relationship among nodes in our derivations. Being mathematically graceful and simple, our analysis provides guideline and theoretical support on system design, simulationbased modeling and performance analysis of the PFS algorithm in the context of cross-layer design. The results are being integrated into the MEMBRANE project [15].

\section{ACKNOWLEDGMENT}

This research was financially supported by grants from EU IST FP6 MEMBRANE project under the contract 027310.

\section{REFERENCES}

[1] F. Kelly, "Charging and Rate Control for Elastic Traffic", Eur. Trans. on Telecommun., February 1997, pp. 33-37.

[2] T. D. Nguye and Y. Han, "A Proportional Fairness Algorithm with QoS Provision in Downlink OFDMA Systems," IEEE Commun Lett., vol. 10, no. 11, November 2006.

[3] H. Seo and B. G. Lee, "A Proportional-Fair Power Allocation Scheme for Fair and Efficient Multiuser OFDM Systems," in Proc. IEEE GLOBECOM, Dallas TX, 2004, pp. 3737-3741.

[4] Z. Han, Z. Ji and K. J. Ray Liu, "Fair Multiuser Channel Allocation for OFDMA Networks Using Nash Bargaining Solutions and Coalitions," IEEE Trans. Commun., vol. 53, no. 8, pp. 1366-1376, August 2005.

[5] A. Jalali, R. Padovani and R. Pankai, "Data Throughput of CDMAHDR: a High Efficiency-High Data Rate Personal Communication Wireless System," in Proc. Veh. Technol. Conf., January. 2001, pp. 5567.

[6] T. Bu, L. Li and R. Ramjee, "Generalized Proportional Fair Scheduling in Third Generation Wireless Data Networks," in Proc. INFOCOM, Barcelona, April 2006, pp. 1-12.

[7] J. M. Holtzman, "Asymptotic analysis of proportional fair algorithm," in Proc. IEEE PIMRC, San Diego, CA, 2001, pp. 33-37.

[8] D. Avidor, S. Mukherjee, J. Ling and C. Papadias, "On Some Properties of the Proportional Fair Scheduling Policy," in Proc. IEEE PIMRC, New Jersey, September 2004, pp. 853-858.

[9] H. J. Kushner and P. A. Whiting, "Asymptotic Properties of Proportional-Fair Sharing Algorithms: Extensions of the Algorithm," in Proc. of the Annual Allerton Conference on Communication, Control and Computing, vol. 41, 2003, pp. 303-311.

[10] J-G. Choi and S. Bahk, "Cell-Throughput Analysis of the Proportional Fair Scheduler in the Single-Cell Environment," IEEE Trans. Veh. Technol., vol. 56, no. 2, pp. 766-778, March 2007.

[11] G. Caire, R. Muller and R. Knopp, "Hard Fairness Versus Proportional Fairness in Wireless Communications: The Single-Cell Case," IEEE Trans. Inf. Theory., vol. 53, no. 4, pp. 1366-1385, April 2007.

[12] S. Bost, "User-level performance of channel-aware scheduling algorithms in wireless data networks," in Proc. INFOCOM, San Francisco, 2003, pp. 321-331.

[13] I. E. Telatar, "Capacity of multi-antenna Gaussian channels," European Transactions on Telecommunications, Vol.10, No.6, pp.585-595, November/December 1999.

[14] P. J. Smith and M. Shafi, "On a Gaussian Approximation to the Capacity of Wireless MIMO Systems," in Proc. IEEE ICC, New York, April 2002, pp. 406-410.

[15] The MEMBRANE website. [Online]. Available: http://www.imperial.ac.uk/MEMBRANE 